\title{
Complement system becomes activated by the classical pathway in intracranial aneurysm walls
}

\author{
Riikka Tulamo ${ }^{1,2}$, Juhana Frösen ${ }^{1,3}$, Sami Junnikkala ${ }^{2}$, Anders Paetau ${ }^{4}$, Marko Kangasniemi ${ }^{5}$, Jose Peláez ${ }^{3}$, \\ Juha Hernesniemi ${ }^{1,3}$, Mika Niemelä ${ }^{1,3}$ and Seppo Meri ${ }^{2}$
}

Inflammation and activation of the complement system in the intracranial aneurysm (IA) wall predispose to IA rupture. We have previously shown that increased C5b-9 accumulation correlates with IA rupture and wall degeneration. To elucidate the underlying mechanisms, we investigated initiators and the pathway of complement activation in unruptured and ruptured IAs. Unruptured and ruptured IA wall samples were studied in parallel sections by immunohistochemical and immunofluorescence stainings for the location and relations of classical and alternative pathway complement components (C1q, C3b/iC3b, C3d, C4b/iC4b; $n=35$ and properdin, $n=10$ ), putative complement activators IgG $(n=90)$, IgM, CRP and OxLDL $(n=10)$, and complement activation endproduct C5b-9. Classical

pathway components were seen in all IAs, and they were located mostly in the extracellular matrix. The early pathway complement components colocalized with each other, but were present in larger areas than C5b-9. The areas positive for complement component accumulation were significantly broader in ruptured than in unruptured IAs. The potential complement activators IgG, IgM, CRP and OxLDL were found mostly in the extracellular matrix and in partial overlap with C5b-9. Lipids were seen in Oil-Red-O staining in colocalization with C5b-9. Complement becomes activated by the classical pathway in the IA wall. The activation appears to be induced by multiple factors, which, in addition to the traditional activators (immunoglobulins, CRP, OxLDL), could involve vascular pressure-induced tissue damage. Despite wide early pathway activation, the terminal pathway is focused on a distinct lipid-rich layer. The profile of the complement components and the association of C $5 b-9$ with lipids in the extracellular matrix indicate a long-term chronic inflammatory process rather than an acute targeted inflammatory reaction. The observed pattern of complement activation may be the consequence of local stress-induced insufficiency of complement regulation in IA walls.

Laboratory Investigation (2010) 90, 168-179; doi:10.1038/labinvest.2009.133; published online 7 December 2009

KEYWORDS: aneurysm; complement; CRP; immunoglobulin; inflammation; oxidized LDL

Saccular intracranial artery aneurysm (IA) rupture causes subarachnoidal hemorrhage (SAH), with up to $50 \%$ mortality. ${ }^{1}$ However, the mechanisms behind IA development, structural weakening and rupture are poorly understood. In a meta-analysis, $2.3 \%$ (range $0.4-6.0 \%$ ) of various populations were found to harbor IA. ${ }^{2}$ Known risk factors for IA rupture and SAH include smoking, hypertension, previous rupture, female gender, excessive alcohol abuse and family history, but these explain only part of the IA disease. ${ }^{3,4}$

Inflammation and its key component complement activation precede IA rupture. Inflammatory cell infiltration and deposits of the activation products of the terminal complement pathway can be found in unruptured IAs. ${ }^{5-9}$ Immunoglobulins and pro-inflammatory cytokines (eg, TNF- $\alpha$ ) have also been detected in human IAs. ${ }^{8,10}$ The IA wall undergoes a remodeling process. This is indicated by the expression of vascular growth factor receptors, proliferation markers, signs of apoptosis and activated intracellular pathways related to growth. ${ }^{11-13}$

The complement system is a major part of innate immunity, which also regulates the adaptive immune system. Growing evidence points to an important role of comple-

\footnotetext{
${ }^{1}$ Neurosurgery Research Group, Biomedicum Helsinki, Helsinki, Finland; ${ }^{2}$ Department of Bacteriology and Immunology, Haartman Institute, University of Helsinki, and HUSLAB, Helsinki University Central Hospital, Helsinki, Finland; ${ }^{3}$ Department of Neurosurgery, Helsinki University Central Hospital, Helsinki, Finland; ${ }^{4}$ Department of Pathology, Haartman Institute, University of Helsinki, and HUSLAB, Helsinki University Central Hospital, Helsinki, Finland and ${ }^{5}$ Medical Imaging Center, Helsinki University Central Hospital, Helsinki, Finland

Correspondence: Dr R Tulamo, MD, Neurosurgery Research Group, Helsinki University Central Hospital, Biomedicum 1, Room B408a2, Haartmaninkatu 8, PO Box 700, Helsinki, Huch FI-00029, Finland.
}

E-mail: riikka.tulamo@hus.fi

Received 3 July 2009; revised 06 October 2009; accepted 11 October 2009 
ment in injury responses, involving the clearance of tissues from accumulating debris together with phagocytes. The complement system consists of approximately 35 proteins, which become activated in a cascade-like manner through sequential proteolytic cleavages and formation of protein complexes. Complement may become activated via the 'classical pathway' in response to endogenous ligands, such as immunoglobulins (eg, IgM or antigen-bound IgG), pentraxins (eg, C-reactive protein (CRP)), dying (apoptotic, ischemic, or necrotic) cells, and extracellular matrix (ECM) proteins, via the 'lectin pathway' by surfaces rich in such oligosaccharides as mannan, or via the 'alternative pathway' in response to specific (bacterial) lipopolysaccharides. A particular role of the alternative pathway is to amplify complement activation in areas lacking sufficient regulatory activity. ${ }^{14}$ Also cholesterol-containing lipids and enzymatically modified or oxidized low-density lipoproteins (OxLDLs), which may accumulate in the walls of stressed blood vessels, for example, in atherosclerosis, are capable of activating complement. In general, complement can recognize foreign particles and alterations in host cell surfaces and structures. If complement activation is not inhibited by membrane-bound or soluble complement inhibitors, it ultimately leads to the formation of the membrane attack complex (MAC), which can cause apoptotic or necrotic death of the target cell. ${ }^{15-17}$

The classical pathway is activated by C1q-mediated recognition of the target. Further activation of this pathway causes $\mathrm{C} 4 \mathrm{~b}$ and its cleavage product $\mathrm{iC} 4 \mathrm{~b}$ to accumulate in the tissues. $\mathrm{C} 4 \mathrm{~b}$ binds to $\mathrm{C} 2$ to generate the $\mathrm{C} 3 / \mathrm{C} 5$ convertase enzyme C4b2a. C3 activation can recruit the alternative pathway and lead to activation of the terminal pathway and the formation of C5b-9 complexes. Alternative pathway activation or spontaneous amplification via $\mathrm{C} 3 / \mathrm{C} 5$ convertases results in the deposition of surface-bound cleavage products $\mathrm{C} 3 \mathrm{~b} / \mathrm{iC} 3 \mathrm{~b}$ and C3d and C5b-9 formation. By being the end product of $\mathrm{C} 3 \mathrm{~b}$ cleavage, $\mathrm{C} 3 \mathrm{~d}$ remains covalently bound to tissues, indicating prolonged complement activation. Anaphylatoxins, the small soluble complement cleavage products C3a and C5a, are chemotactic to and capable of activating inflammatory cells, whereas the target-bound complement components opsonize the target for phagocytic cells. ${ }^{15,16}$

We have previously shown that C5b-9 accumulates in both unruptured and ruptured IA walls. ${ }^{9}$ Ruptured IAs harbor heavier C5b-9 accumulation and C5b-9 accumulation correlates with increased inflammatory cell infiltration, aneurysm wall degeneration and rupture. ${ }^{9}$ As the origin and causes of complement activation in IAs are unknown, we studied the complement activation pathways and potential activators in both unruptured and ruptured IAs. Our objectives were to determine whether the complement becomes activated in IAs by specific activators or by its general activity in recognizing nonviable tissue components, and whether the nature of complement activation differs between unruptured and ruptured IAs. Activation of this important innate defense mechanism may provide a link between mechanical stressinduced tissue damage and inflammation-induced weakening of the IA wall.

\section{PATIENTS AND METHODS Sample and Clinical Data Collection}

To study the complement pathways and their activation, we used a series (total $n=100$ ) of human saccular IA domes resected intraoperatively after clipping at the Department of Neurosurgery, Helsinki University Central Hospital. The specimens were immediately snap-frozen in liquid nitrogen and stored at $-80^{\circ} \mathrm{C}$. Clinical data (Table 1) were collected from medical records, and IA dimensions were obtained from the preoperative computed tomography angiography images. The study protocol was approved by the local ethics committee.

\section{Primary Antibodies}

The antibodies and some of their properties are listed in Table 2. The presence of C1q, the first component of complement classical pathway, was detected with Dako's antibody that recognizes both the collagenous and globular parts of the C1q molecule. Activation of the subsequent step in the classical complement pathway was detected by Dako's antibody raised against the $\mathrm{C} 4 \mathrm{c}$ fragment that recognizes $\mathrm{C} 4, \mathrm{C} 4 \mathrm{~b}$ as well as $\mathrm{iC} 4 \mathrm{~b}$, the split product of $\mathrm{C} 4 \mathrm{~b}$. Of these, $\mathrm{C} 4 \mathrm{~b}$ and $\mathrm{iC} 4 \mathrm{~b}$ are recognized in a vascular tissue by the antibody because they remain firmly bound to their targets via a covalent linkage. Properdin, detected with an antibody by Incstar, is an accelerator of the alternative pathway and a marker of independent alternative pathway activation because it binds strongly to active $\mathrm{C} 3 \mathrm{bBb}$ convertases. The alternative pathway activation and amplification product $\mathrm{C} 3 \mathrm{~b}$ and its cleavage fragment iC3b were detected with Dako's antibody raised against $\mathrm{C} 3 \mathrm{c}$ that recognizes both of these covalently bound C3 activation products in vascular tissue. C3d was stained by a monoclonal antibody. It recognizes the $\mathrm{C} 3 \mathrm{~d}$ fragment that carries the binding site for covalent linkage in C3b, iC3b as well as in the separate C3dg fragment that remains surfacebound after release of the $\mathrm{C} 3 \mathrm{c}$ fragment. The terminal complement pathway activation and associated terminal complement complex formation was detected by Quidel's antibody against SC5b-9. The antibody does not discriminate between soluble S-protein-coupled SC5b-9 or target-bound C5b-9 (MAC) complexes, but shows the local terminal pathway activation because the diffusion of the C5b-9 complex within a tissue is limited due to its firm binding to tissue structures or insertion into target membranes. As a control, the immunohistochemical localization of C5b-9 complex within the aneurysm tissue was also verified with another monoclonal antibody $\mathrm{Wu}$ 7-2 raised against SC5b-9, a kind gift from professor Reinhard Würzner, Innsbruck, Austria $^{18}$ (Supplementary Figure 1). Tissue-associated LDL particles were detected by two antibodies raised against 
Table 1 Distribution of clinical variables related to intracranial artery aneurysms studied for complement activation

\begin{tabular}{|c|c|c|c|}
\hline & \multicolumn{2}{|c|}{ Patients with } & \multirow[b]{2}{*}{$P$-value ${ }^{a}$} \\
\hline & Unruptured IAs & Ruptured IAs & \\
\hline Aneurysms studied for complement ( $n$ ) & 26 & 19 & \\
\hline Patient age (years) ${ }^{b}$ & $55(39-71)$ & $54(13-75)$ & 0.924 \\
\hline Females $^{c}$ & $65 \%(17 / 26)$ & $44 \%(8 / 18)$ & 0.160 \\
\hline Patients with previous $\mathrm{SAH}^{\mathrm{c}}$ & $12 \%(3 / 26)$ & $0 \%(0 / 18)$ & 0.080 \\
\hline IA neck diameter $(\mathrm{mm})^{\mathrm{b}}$ & $4(2-10)$ & $4(2-5)$ & 0.350 \\
\hline IA fundus length $(\mathrm{mm})^{\mathrm{b}}$ & $8(3-29)$ & $9(6-14)$ & 0.162 \\
\hline IA fundus width $(\mathrm{mm})^{\mathrm{b}}$ & $6(3-34)$ & $7(3-12)$ & 0.121 \\
\hline Patient age (years) ${ }^{b}$ & $54(28-71)$ & $54(28-84)$ & 0.384 \\
\hline Females $^{c}$ & $61 \%(22$ of 36$)$ & $57 \%$ (30 of 53 ) & 0.546 \\
\hline Patients with multiple $(\geq 2) \operatorname{las}^{c}$ & $41 \%(14$ of 34$)$ & $33 \%(16$ of 48$)$ & 0.497 \\
\hline Patients with previous $\mathrm{SAH}^{\mathrm{c}}$ & $21 \%(7$ of 34$)$ & $4 \%(2$ of 49$)$ & 0.047 \\
\hline IA neck diameter $(\mathrm{mm})^{\mathrm{b}}$ & $4(2-10)$ & $4(2-10)$ & 0.689 \\
\hline IA fundus length $(\mathrm{mm})^{\mathrm{b}}$ & $7(3-29)$ & $8(4-17)$ & 0.056 \\
\hline IA fundus width $(\mathrm{mm})^{\mathrm{b}}$ & $6(3-34)$ & $7(2-15)$ & 0.134 \\
\hline Time from IA rupture to sample resection & & $22 \mathrm{~h}(4 \mathrm{~h}-48 \mathrm{~d})$ & \\
\hline
\end{tabular}

IA, intracranial aneurysm.

${ }^{a} P$-value $<0.05$ considered significant.

${ }^{\mathrm{b}}$ Mann-Whitney $U$-test used, median and range are given for continuous variables.

${ }^{c} \chi^{2}$-test used, proportions are given.

differentially oxidized LDL as described in Holvoet $e t a l^{19}$ and Palinski et al. ${ }^{20}$

\section{Immunohistochemistry}

For immunohistochemistry, samples were cryosectioned at $4 \mu \mathrm{m}$. The sections were fixed with $4 \%$ paraformaldehyde, and non-specific binding was blocked with 3-5\% normal goat or horse serum (Vector Laboratories, Burlingame, CA, USA) in phosphate-buffered saline (PBS). Some sections were additionally blocked with $0.5 \%$ cold fish skin gelatin (Sigma) in PBS containing $0.5 \%$ bovine serum albumin (Sigma-Aldrich, St Louis, MO, USA) (BSA/PBS). The primary antibodies against complement components or OxLDL (Table 2) were diluted in $1 \%$ BSA/PBS and incubated for $30 \mathrm{~min}$ at room temperature or overnight at $+4^{\circ} \mathrm{C}$. The endogenous peroxidase was blocked with $0.3-0.7 \%$ hydrogen peroxide in PBS or methanol. The sections were incubated with biotinylated secondary antibodies (Vector) for $30 \mathrm{~min}$ at room temperature. The signal was enhanced by horseradish peroxidase or alkaline phosphatase-conjugated avidin-biotin complexes and visualized with DAB (Sigma) or Vector Blue (Vector), respectively. The background was stained with Mayer's hematoxylin or Carmaleum. Human tonsil served as a positive control for the stainings. For negative controls, the primary antibody was omitted or replaced with an irrelevant antibody of the same immunoglobulin isotype as the primary antibody.

\section{Immunofluorescence Stainings}

For immunoglobulin, CRP, $\alpha$-smooth muscle actin ( $\alpha \mathrm{SMA})$, properdin, and C5b-9 stainings, we used the indirect immunofluorescence method. Fixation, blocking, and incubation with the primary antibody were performed as described above. The primary antibody (Table 2), if unconjugated, was detected with fluorochrome-conjugated Alexa Fluor 488 (green) or 546 (red) secondary antibody (Molecular Probes, Eugene, OR, USA) diluted to 1:100-1:200. For doublestainings, the incubations were repeated with antibodies originating from different species. The slides were mounted in Vectashield with DAPI (Vector) to detect the nuclei. 


\section{Oil-Red-O Staining}

Lipids were stained with Oil-Red-O in unfixed cryosections. The sections were incubated in absolute propylene glycol (Sigma) for $5 \mathrm{~min}$, followed by incubation in $0.5 \%$ Oil-Red$\mathrm{O}$ solution in propylene glycol for $7 \mathrm{~min}$, washed twice in $85 \%$ propylene glycol, running tap water, and aqua. The nuclei were background-stained with Mayer's hematoxylin.

\section{Imaging and Histological Analysis}

The immunohistochemical stainings were photomicrographed with an Axioplan 2 imaging microscope and AxioCam MRc (Carl Zeiss Vision GmbH, Aalen, Germany). The immunofluorescence stainings were viewed and photographed with Axioplan 2 and AxioCam (HR/MR), with appropriate filters to create pseudocolored images (Zeiss). The pseudocolored images are presented in figures within the Results. For separate channel images, please see the Supplementary Figures 2 and 3.

Serial sections of 35 IAs (16 unruptured, 19 ruptured) were stained for complement components C1q, C3b/iC3b, C3d, $\mathrm{C} 4 \mathrm{~b} / \mathrm{iC} 4 \mathrm{~b}$, and C5b-9. Antigen deposition in the IA wall was classified as mild, moderate, or strong depending on the extent of the positively staining area relative to the whole IA wall surface area (mild $<1 / 3$, moderate $1 / 3-2 / 3$, strong $>2 / 3$ ). The presence or absence of complement components was screened in the areas of interest within parallel sections. Another series of 10 single- and double-stained unruptured
IAs was screened for the location of the positive staining for the potential complement activators, complement components (properdin, C1q, and C5b-9), and lipids within the cells or ECM. Multiple antibodies against OxLDL were used to show the difference in the level and type of oxidative modification. The localizations of the antigens were compared with each other in the serial sections. An enlarged series of 90 IAs (36 unruptured, 54 ruptured) was used to evaluate the presence or absence of IgG and its location within the cells or ECM of the IA wall.

\section{Statistical Analysis}

The clinical and radiological data and the data from immunostainings were compared with the IA rupture status. Medians and ranges were calculated, and $\chi^{2}$ or MannWhitney $U$-tests were used for categorical and continuous variables, respectively (SPSS 16.0, SPSS, Chicago, IL, USA). The $\alpha$ level was set at 0.05 .

\section{RESULTS \\ Patients and Aneurysms}

The clinical and radiological data of the patients and aneurysms are presented in Table 1. No significant differences in smoking status, family history, or hypertension between patients with ruptured and those with unruptured aneurysms were observed (data not shown).

Table 2 Primary antibodies used in immunohistochemical and immunofluorescence stainings ${ }^{a}$

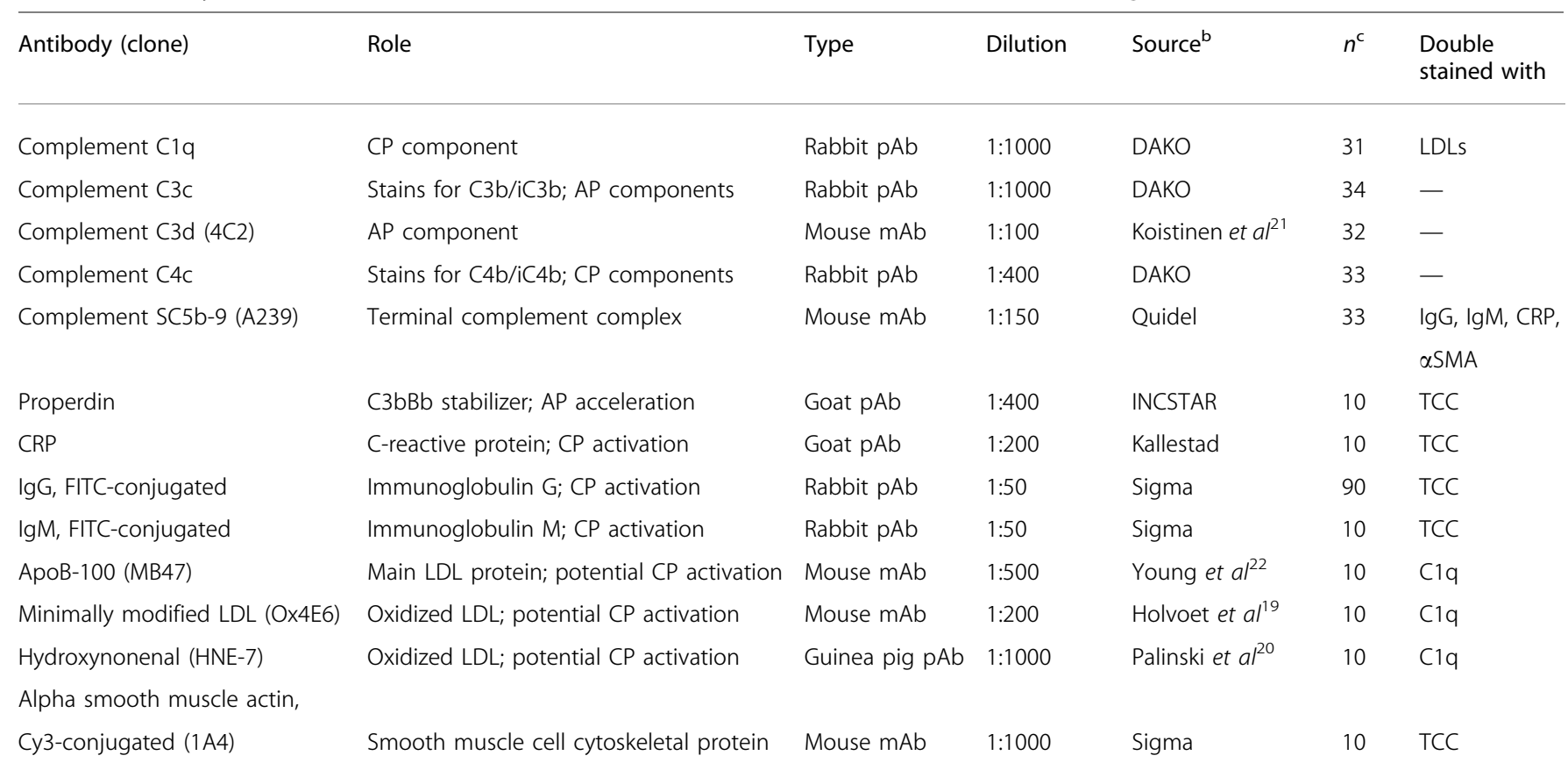

\footnotetext{
${ }^{\mathrm{a}} \mathrm{CP}$, complement classical pathway; AP, complement alternative pathway; LDL, low-density lipoprotein; pAb, polyclonal antibody; mAb, monoclonal antibody; TCC, terminal complement complex; $\alpha$ SMA, alpha smooth muscle actin.

bDAKO, DakoCytomation, Glostrup, Denmark; Quidel, Quidel Corporation, San Diego, CA, USA; INCSTAR Corporation, Stillwater, MN, USA; Kallestad, Chaska, MI, USA; Sigma, Sigma-Aldrich, St Louis, MO, USA.

${ }^{\mathrm{C}}$ Number of IAs studied with the antibody.
} 

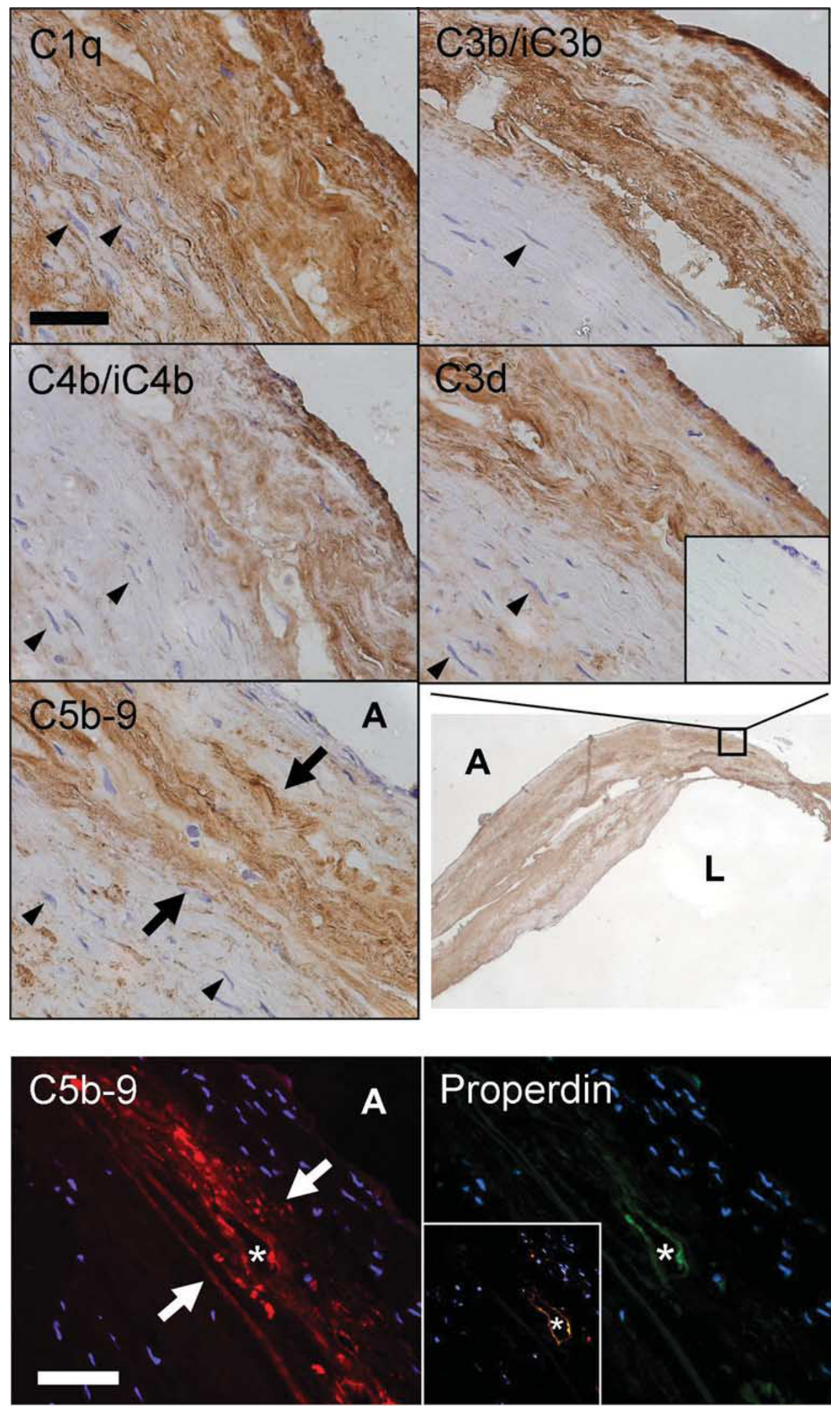


\section{C1q, First Component of the Classical Pathway}

C1q became stained in large areas throughout the IA walls. Clq deposition was located mostly in the ECM. Some of the positive staining was seen in organized ECM structures, for example, collagen fibrils, and in individual cells. C1q staining also covered those areas that were positive for other complement components (Figure 1). By determining the area of positive staining, C1q accumulation was found to occur in wider areas in ruptured than unruptured IAs $(P=0.011)$ (Figure 2).

\section{C4b/ic4b, Activation Products of the Classical Pathway}

After initiation of the classical pathway, the $\mathrm{C} 1 \mathrm{~s}$ component of $\mathrm{C} 1$ activates $\mathrm{C} 4$ to $\mathrm{C} 4 \mathrm{~b}$. The antibody to $\mathrm{C} 4$ recognizes $\mathrm{C} 4 \mathrm{~b}$ as well as its inactivation product $\mathrm{iC} 4 \mathrm{~b}$, both of which become covalently bound to targets. In IAs, $\mathrm{C} 4 \mathrm{~b} / \mathrm{iC} 4 \mathrm{~b}$ was seen in the ECM in areas positive for $\mathrm{Clq}$ and for C5b-9. The maximum positive staining was seen in a band-like area in the outer IA wall (Figure 1). Ruptured IAs showed a broader accumulation of $\mathrm{C} 4 \mathrm{~b} / \mathrm{iC} 4 \mathrm{~b}$ than unruptured IAs $(P=0.001)$ (Figure 2).

\section{C3 Cleavage Products C3b/ic3b and C3d}

$\mathrm{C} 3 \mathrm{~b} / \mathrm{iC} 3 \mathrm{~b}$ and $\mathrm{C} 3 \mathrm{~d}$ were detected in similar areas, but the positive staining for $\mathrm{C} 3 \mathrm{~d}$ was more restricted than that for C3b/iC3b. C3 activation covered also areas that were positive for $\mathrm{C} 4 \mathrm{~b} / \mathrm{iC} 4 \mathrm{~b}$ and $\mathrm{C} 5 \mathrm{~b}-9$. The maximum positive staining was seen in a band-like area in the outer IA wall (Figure 1). The accumulation of $\mathrm{C} 3 \mathrm{~b} / \mathrm{iC} 3 \mathrm{~b}$ and $\mathrm{C} 3 \mathrm{~d}$ was heavier in ruptured than unruptured IAs $(P<0.001$ and $P=0.048$, respectively) (Figure 2).

\section{Properdin, Indicator of the Alternative Pathway}

The alternative pathway C3 convertase $(\mathrm{C} 3 \mathrm{bBb})$ stabilizer, properdin, was occasionally seen bound to the ECM, mainly in sparse patches in areas other than the maximum C5b-9 accumulation (Figure 1). Occasionally some overlapping with C5b-9 was seen. In 4/10 unruptured IAs separate properdin-positive cells were also noted.

\section{Terminal Complement Complex C5b-9}

The end product of complement activation, C5b-9, became stained in most IAs as a band-like layer located mainly in the outer part of the IA wall, as we have reported previously. ${ }^{9}$ C5b-9 was located in the same areas, but to a lesser degree than the above-mentioned complement components (Figure 1).

\section{Complement Components and Smooth Muscle Cells}

The areas with band-like staining patterns for C5b-9 in the outer parts of the IA wall mostly lacked nuclei (Figure 1). Some of the scattered C5b-9 was located in the inner wall in areas rich in smooth muscle cells (SMCs) (Figure 3a). $\alpha$ SMA and C5b-9 double-positive cells were seen in only $1 / 10$ unruptured IA samples (Figure 3b), and the C5b-9-positive cells were seen in other $4 / 10$ samples.

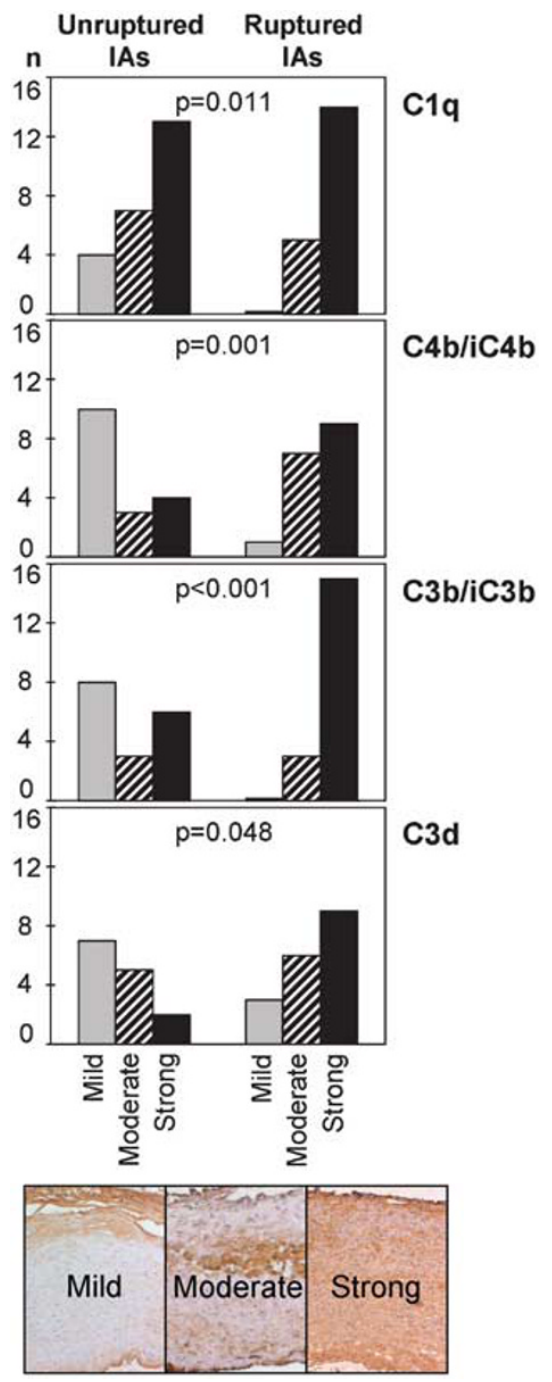

Figure 2 Distribution of mild, moderate, and strong staining for complement components in unruptured and ruptured intracranial aneurysms. $\chi^{2}$-test; $P<0.05$ is considered to indicate a significant difference. The photographs give examples of mild, moderate, and strong staining patterns of $\mathrm{C} 4 \mathrm{~b} / \mathrm{iC} 4 \mathrm{~b}$ in the aneurysm wall.

Figure $1 \mathrm{Immunohistochemical} \mathrm{and} \mathrm{immunofluorescence} \mathrm{staining} \mathrm{for} \mathrm{complement} \mathrm{components} \mathrm{in} \mathrm{parallel} \mathrm{sections} \mathrm{of} \mathrm{an} \mathrm{area} \mathrm{of} \mathrm{the} \mathrm{wall} \mathrm{of} \mathrm{a} \mathrm{ruptured}$ (immunohistochemical stainings) and an unruptured (immunofluorescent stainings) intracranial aneurysm. Both classical and alternative pathway components were detected in the extracellular matrix but only classical pathway components in the same degenerated band-like area with strong $\mathrm{C} 5 \mathrm{~b}-9$ staining that lacks nuclei (arrows). The intramural cells (arrowheads) remained negative for complement. C3d was seen in subareas of C3b/iC3b staining. The unruptured aneurysm has some autofluorescent atheromatous calcifications (asterisk). $\mathrm{L}$ and $\mathrm{A}$ indicate luminal and adventitial sides of the aneurysm wall, respectively. The insets show negative controls (primary antibody omitted). Scale bar corresponds to $50 \mu \mathrm{m}$. 

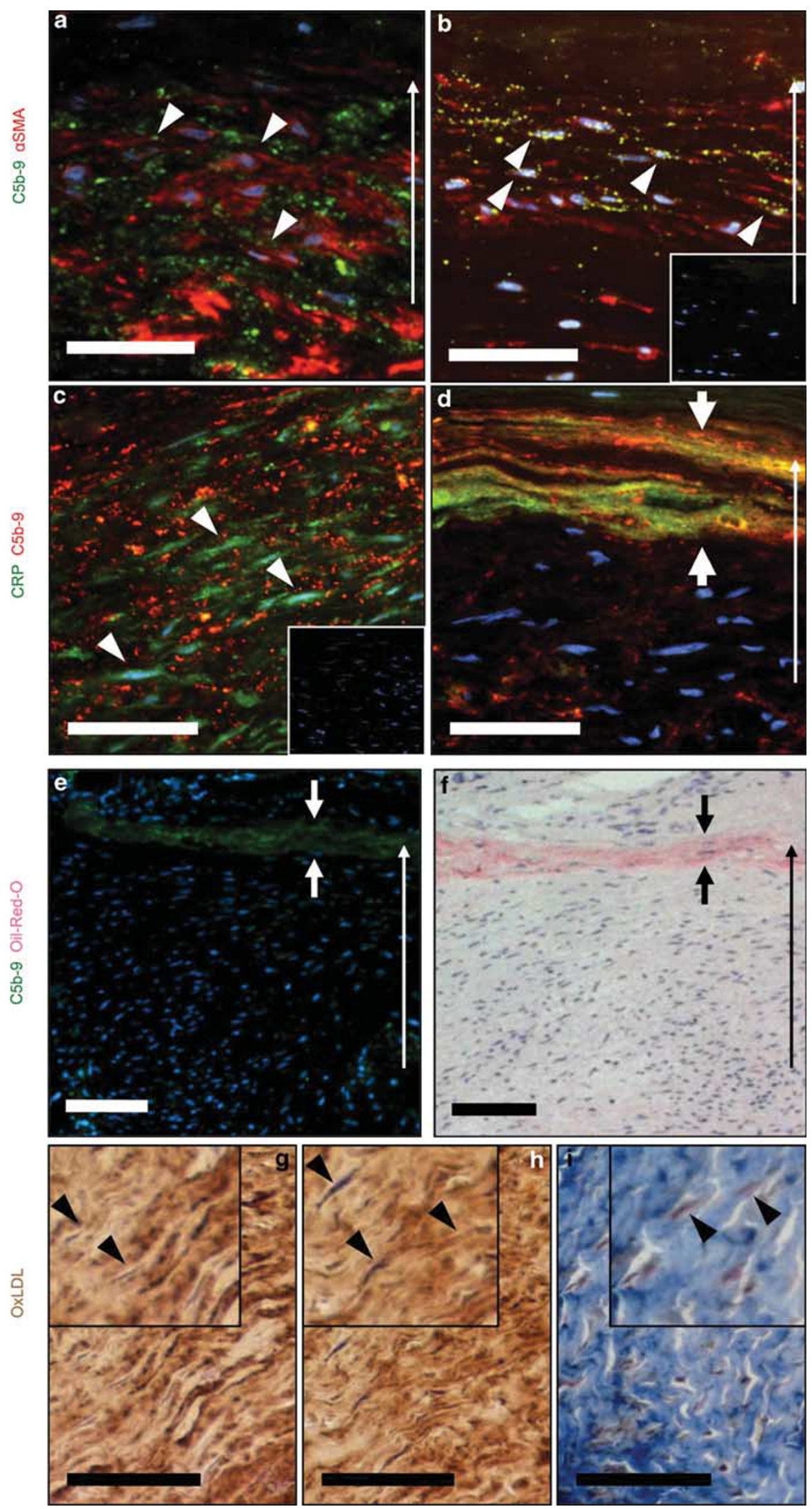


\section{C-Reactive Protein}

CRP is an acute phase-protein that can bind to injured tissues and activate the classical pathway. Positive staining for CRP was seen mainly in the ECM. In 5/10 unruptured IAs, a few CRP-positive cells were also seen; in one IA sample, almost all of the mural cells stained positive for CRP (Figure 3c). In double-stainings with C5b-9, only occasional double-positive cells were detected. Instead, the ECM showed faint double positivity for CRP and C5b-9. CRP was extensively positively stained in the band-like area of heavy C5b-9 staining in the outer IA wall (Figure 3d).

\section{Lipids and Oxidized Low-Density Lipoproteins}

Neutral triglycerides and lipids seen in Oil-Red-O staining were located in the same areas as C5b-9, whereas OxLDL showed positive staining throughout the IA wall (Figure $3 \mathrm{e}$ and $\mathrm{f}$ ). The ECM stained positively in all samples for the main LDL protein ApoB-100 and for minimally modified LDL (Figure $3 \mathrm{~g}$ and $\mathrm{h}$ ). The positive staining for cells was seen with ApoB-100, minimally modified LDL and hydroxynonenal in $6 / 10,8 / 10$, and $10 / 10$ samples, respectively. In double-stainings, the areas positive for both $\mathrm{Clq}$ and different OxLDLs overlapped, but lacked a clear double-staining pattern (Figure 3i).

\section{$\lg G$}

IgG was detected in 61/90 IA walls (68\%). Of the IgG-positive and -negative samples, 38/61 (62\%) and 16/29 (55\%) were ruptured, respectively $(P=0.520)$. IgG was located diffusely in the ECM (Figure 4a) of 57/61 samples (93\%). IgG-positive cells (Figure 4e) were detected in $7 / 36$ unruptured $(19 \%)$ and $10 / 54$ ruptured IAs $(19 \%)(P=0.913)$. In some IAs, IgG homogeneously stained the whole ECM, whereas in others the positive staining faded gradually from the lumen to the outer wall (Figure 4e). No distinct patterns of IgG binding suggesting specific antigenic targets were seen. In 10 unruptured IAs, some overlap between the IgG- and C5b-9positive areas was present, but no double-staining could be detected (Figure 4c).

\section{IgM}

Of the 10 unruptured IAs, eight stained positive for IgM. IgM was located mainly in the ECM in a patch-like manner. Occasionally, a gradually decreasing staining from the lumen to the outer wall was seen (Figure $4 \mathrm{~d}$ ). In one sample, an additional IgM deposit in the ECM just below a vas vasorum was seen (Figure 4f). In two samples, a few IgM-positive cells were detected. Some overlap of the IgM and C5b-9 was present in the ECM, especially in the areas of band-like heavy accumulation of C5b-9 in the outer wall (Figure 4b). IgM was located in subareas of regions positive for IgG (Figure $4 a-d$ ).

\section{DISCUSSION}

We have previously shown that terminal pathway complement activation is associated with intracranial aneurysm rupture and wall degeneration. ${ }^{9}$ In this study, we investigated the pathways and activators by which the complement system in the IA wall can become activated. We found that components of the classical pathway and also all of the potential classical pathway activators studied, that is, IgG, IgM, CRP, and OxLDLs, were present in the IA wall. The major lipid accumulation colocalized with C5b-9. Complement activation was more pronounced in ruptured than unruptured IAs, consistent with our earlier observations for C5b-9. These results indicate that complement becomes activated broadly via the classical pathway and leads to deposition of C5b-9 in distinct lipid-rich areas of the IA wall. We postulate that physicochemical stress in the intracranial arteries leads to deposition and exposure of specific complement-activating factors in the IA wall, predisposing it to rupture.

\section{Immunoglobulins and Complement Activation}

IgM and antigen-bound IgG subclasses IgG1 and IgG3 are potent classical pathway activators, and naturally occurring IgM antibodies have been proposed to activate the classical pathway, for example, in myocardial infarction. ${ }^{23}$ In our series, IgG as well as IgM were seen in the IA wall, mostly bound to the ECM. This finding is consistent with previous results of immunoglobulin accumulation in abnormal vascular tissues; abdominal aortic aneurysms show strongly increased amounts of IgG together with complement C3d. ${ }^{24}$ Immunoglobulins have been detected earlier in unruptured IA walls in similar proportions as those seen here in both unruptured and ruptured IAs, but not in control basilar artery samples. ${ }^{8}$ In atherosclerotic coronary arteries, IgM has been shown to accumulate and colocalize with the classical pathway activation products $\mathrm{C} 4 \mathrm{~b} / \mathrm{iC} 4 \mathrm{~b}$ in the superficial intima. $^{25}$

We noted a gradually decreasing staining for $\operatorname{IgG}$ in the lumen-to-adventitia direction, ECM-bound IgG and IgM, and an incidental IgM accumulation next to a vas vasorum. De-endothelialization occurs during IA progression and is seen in both human and experimental animal IAs. ${ }^{5-7,26}$ Heavier C5b-9 accumulations have been observed in

Figure 3 Immunofluorescence staining for C5b-9 (green) and smooth muscle cells (red) (a and b), for C-reactive protein (CRP) (green) and C5b-9 (red) (c and d), and for C5b-9 (green) and lipids in Oil-Red-O (red) (e and f) and immunohistochemical staining for oxidized low-density lipoproteins (OxLDLs) (brown; g-i) and C1q (blue; i) on unruptured intracranial aneurysms. Double positivity in immunofluorescence stainings is shown in yellow. Arrowheads point to $\alpha$-smooth muscle actin ( $\alpha$ SMA) (a and $\mathbf{b})$ and CRP (c). Arrows show the C5b-9-positive degenerated band-like area in the outer aneurysm wall (d and e) that contains neutral lipids (f). Arrowheads (g-i) point to cells positive for differentially oxidized low-density lipoproteins: ApoB-100 (g), minimally modified LDL (h), and hydroxynonenal (i). The slim arrows indicate the lumen-to-adventitia direction. The insets (b and $\mathbf{c}$ ) are negative controls (primary antibody omitted). Scale bar corresponds to $50 \mu \mathrm{m}$. 
$\lg G$
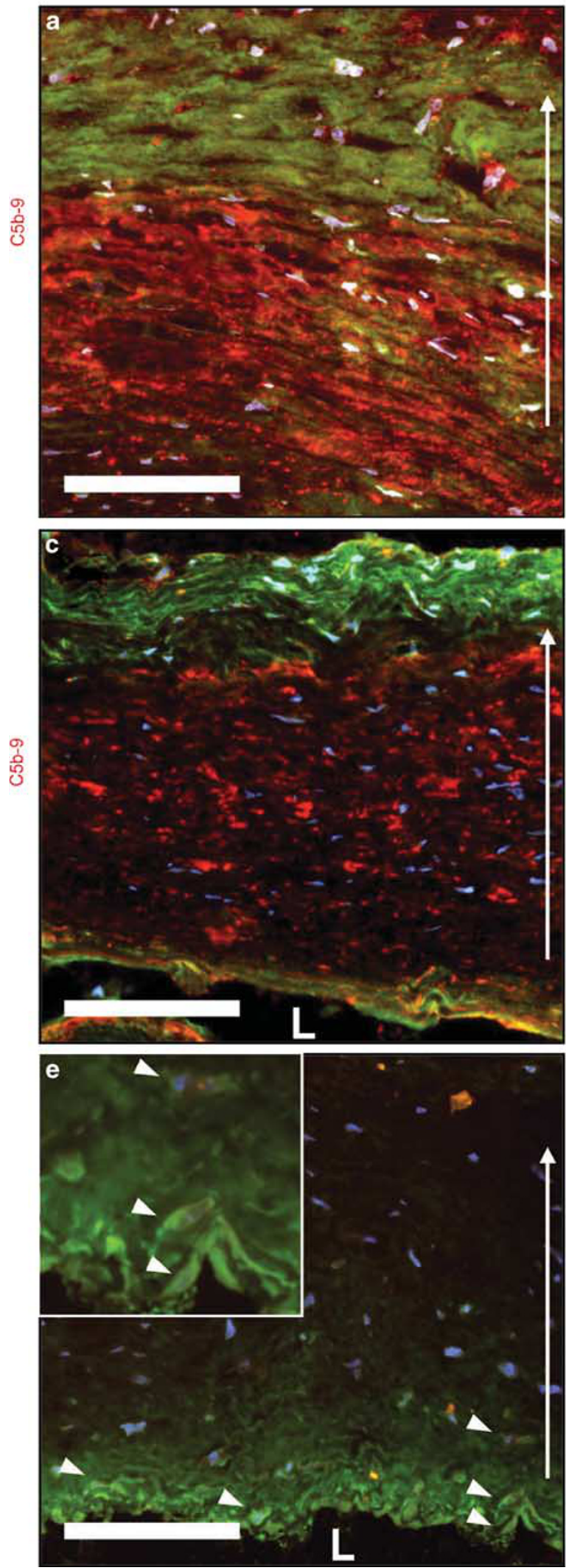

$\lg M$
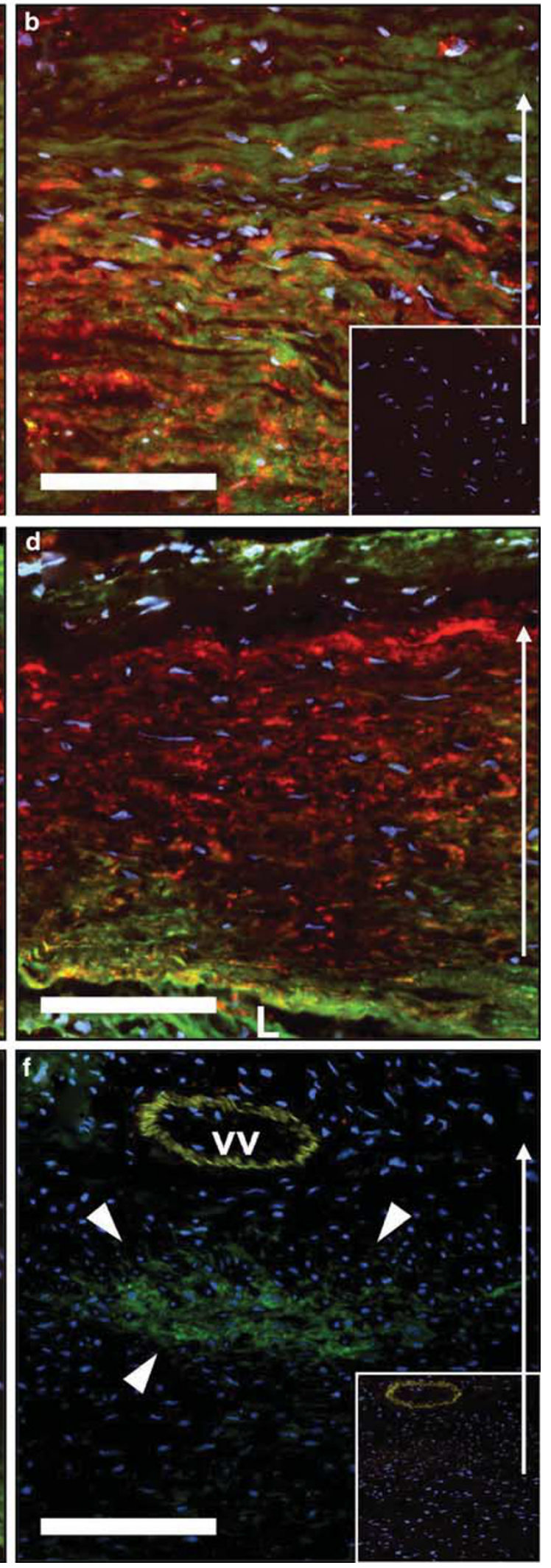
de-endothelialized IAs. ${ }^{9}$ Normal centrifugal mass transport of micro- and macromolecules through the vascular wall because of hemodynamic forces and their clearance is disturbed in pathological conditions. ${ }^{27}$ Thus, we hypothesize that immunoglobulins, as well as complement components, may leak and accumulate in the IA wall because of endothelial dysfunction and impaired clearance mechanisms. Any antibodies with natural or acquired specificity for structures in the ECM would be expected to enhance immunoglobulin deposition. Novel antigen exposure could occur via pressure-induced damage and/or proteolysis of components of the ECM.

\section{Role of OxLDL and CRP in Complement Activation in the IA Wall}

We found lipid accumulation in the same areas where C5b-9 was deposited. We also detected OxLDLs in areas positive for CRP, similarly as seen in atherosclerotic plaques. ${ }^{28}$ Native and oxidized lipids accumulate in stressed vascular walls and oxidized lipids may activate the classical pathway in vivo through Clq in the presence of CRP. ${ }^{17,29}$ Native CRP is present widely in inflamed tissues, but only in a few healthy aortas. $^{30-32} \mathrm{C} 1 \mathrm{q}$ was mostly bound to ECM, suggesting that classical pathway activation in IA walls could be induced by OxLDL and/or CRP. However, C1q is also capable of interacting with several matrix proteins. ${ }^{16}$ Surface-bound CRP itself is a potent complement classical pathway activator. In one unruptured IA, we found many CRP-positive mural cells without C5b-9 accumulation, which might be explained by CRP's need to be modified for classical pathway activation or by its ability to bind complement inhibitors, for example, factor $\mathrm{H}$, to protect the target structures from further complement activation. We hypothesize that the lipid accumulation in the outer wall may be due to impaired clearance mechanisms and lead to an assembly of the terminal complement complex C5b-9. Whether the formed complex is a fully assembled MAC with polymeric C9 or a terminal complex without poly $\mathrm{C} 9$ cannot be assessed at this point.

\section{Classical vs Alternative Pathway Activation in the IA Wall}

The presence of $\mathrm{C} 4 \mathrm{~b} / \mathrm{iC} 4 \mathrm{~b}$ together with $\mathrm{Clq}$ in our series clearly indicates that the classical pathway of complement has become activated in IA walls. Activation was seen in areas with C5b-9 deposition. Properdin, a specific marker for alternative pathway activation was only weakly seen in the IA walls. This suggests the role of the classical pathway in the initiation of complement activation, eventually leading to C5b-9 formation. Complement C3b/iC3b deposits were also observed in the same areas and usually in more abundance than $\mathrm{C} 4 \mathrm{~b} / \mathrm{iC} 4 \mathrm{~b}$. The alternative pathway may thus become recruited in inflamed tissue following persistent classical pathway activation. This may be due to the action of the C3/C5 convertases and alternative pathway amplification. ${ }^{15,16}$ The deposition of C3d, a covalently bound long-lived breakdown product of $\mathrm{C} 3 \mathrm{~b}$, thus suggests more prolonged inflammation and complement accumulation. ${ }^{33,34}$ The presence of $\mathrm{C} 4 \mathrm{~b} / \mathrm{iC} 4 \mathrm{~b}$ in the same areas with $\mathrm{C} 3 \mathrm{~b} / \mathrm{iC} 3 \mathrm{~b}$ and $\mathrm{C} 3 \mathrm{~d}$ may indicate that classical pathway activation is involved in the initiation of alternative pathway amplification in the IA wall.

\section{Role of Complement Activation in IA Wall Cell Loss}

Important structural and functional components of the IA wall are SMCs, which disappear in IA wall degeneration because of apoptotic and necrotic cell death. ${ }^{7,9,35,36}$ In this series, we managed to detect C5b-9-positive SMCs in one unruptured IA and a few C5b-9-positive cells in 4/10 other unruptured IAs. Earlier, however, we were unable to find C5b-9-positive apoptotic cells. ${ }^{9}$ Although the complement system would be capable of inducing cell death in the IA wall, a massive attack against SMCs by the MAC appears an unlikely explanation for the loss of cells in the IA wall.

\section{Complement Activation and IA Rupture}

In addition to a preceding low-grade complement activation, the rupture itself and the following SAH may induce complement activation in the IA wall. However, the following facts indicate that the complement activation seen in ruptured IAs has occurred already before the rupture. First, the unruptured IAs also show relatively heavy accumulation of $\mathrm{C} 3 \mathrm{~b} / \mathrm{iC} 3 \mathrm{~b}$ and C3d. Second, the magnitude of C5b-9 accumulation in the IA wall is independent of the time from rupture to sample collection and is seen also in all unruptured IAs. ${ }^{9}$ Third, the heavier inflammatory cell infiltrations into the ruptured IA wall have occurred before the rupture. ${ }^{6,7}$ The fact that complement activation products accumulate in unruptured IAs does not preclude their contribution to ruptures.

\section{Is Complement Activation in the IA Wall Due to Impaired Clearance?}

The IA wall is exposed to both mechanical hemodynamic stresses and humoral inflammatory stresses. Disturbances and possible overloading in the wall clearance mechanisms may lead to the accumulation of molecules and material in the vascular wall that normally would be transported through it. ${ }^{27}$ We found terminal complement complex accumulation

Figure 4 Immunofluorescence staining for the relations of $\operatorname{lgG}$ (green; a, c, and e), IgM (green; b, d, and f), and C5b-9 (red; a-d) in two unruptured intracranial aneurysms (a-e and $\mathbf{f}$ ) on degenerated (a and $\mathbf{b}$ ) and cellular (c-f) parts of the wall. Arrowheads point to lgG-positive cells (e) and lgM accumulation (f) close to a vas vasorum (vv) whose elastic lamina shows some autofluorescence. The slim arrows indicate the lumen-to-adventitia direction. $\mathrm{L}$ indicates lumen. The insets (b and $\mathbf{f}$ ) are negative controls (primary antibody omitted). Scale bar corresponds to $100 \mu \mathrm{m}$. 
with lipids in the outer part of the wall. Complement deposits might thus serve as a marker of long-term stress of the IA wall, an attempt to remove debris, or disturbed complement regulation in the ECM. ${ }^{16}$ Based on the lack of 1:1 colocalization of complement components and potential complement activators, complement seems to have become activated in the IA wall in response to more than one specific activator. Complement activation in the IA wall might thus be caused by the combined effect of traditional activators plus mechanochemical stress, leading to a disturbance in the local tissue homeostasis.

\section{Conclusions and Clinical Relevance}

As complement activation in the IA wall is initiated by the classical pathway, but is not clearly associated with IgG, IgM, OxLDL, or CRP, it likely is caused by a combined effect of the traditional activators and a more general change in the local tissue environment. Accumulation of complement components in the ECM indicates a long-term chronic inflammatory process rather than an acute antigentargeted immune attack or inflammation. The observed complement activation may be the consequence of increased physical pressure, leading to exposure of targets for both antibodies and initiators in innate immune reactions. This could occur in combination with local stress-induced insufficiency in complement regulation. Therefore, our results warrant further studies on complement regulation in the IA wall. Better understanding of the pathophysiology behind IA rupture will facilitate the development of novel therapeutic options.

Supplementary Information accompanies the paper on the Laboratory Investigation website (http://www.laboratoryinvestigation.org)

\section{ACKNOWLEDGEMENTS}

We thank Professor Seppo Ylä-Herttuala for his kind gift of anti-OxLDL antibodies; Professor Reinhard Würzner for his kind gift of antibody Wu 7-2; Ilse Lappalainen, Tanja Eriksson, and Taru Puhakka for technical assistance; RN Maarit Alalahti for her help in sample collection; and MD Monsoor Foroughi for language revision. This work was supported by Helsinki University Central Hospital EVO Grants TYH 4204, 5207, 7207, and 7214; the Maire Taponen Foundation, Helsinki, Finland; the Biomedicum Helsinki Foundation, Helsinki, Finland; the Orion-Farmos Foundation, Espoo, Finland; the Academy of Finland, Helsinki, Finland; and NIH Grant EY 11515.

\section{DISCLOSURE/CONFLICT OF INTEREST}

The authors declare no conflict of interest.

1. van Gijn J, Rinkel GJ. Subarachnoid haemorrhage: diagnosis, causes and management. Brain 2001;124:249-278.

2. Rinkel GJ, Djibuti M, Algra A, et al. Prevalence and risk of rupture of intracranial aneurysms: a systematic review. Stroke 1998;29:251-256.

3. Juvela S, Porras M, Poussa K. Natural history of unruptured intracranial aneurysms: probability of and risk factors for aneurysm rupture. J Neurosurg 2000;93:379-387.

4. Ishibashi T, Murayama $Y$, Urashima $M$, et al. Unruptured intracranial aneurysms: incidence of rupture and risk factors. Stroke 2009;40: 313-316.
5. Crompton MR. Mechanism of growth and rupture in cerebral berry aneurysms. Br Med J 1966;1:1138-1142.

6. Kataoka K, Taneda M, Asai T, et al. Structural fragility and inflammatory response of ruptured cerebral aneurysms. A comparative study between ruptured and unruptured cerebral aneurysms. Stroke 1999;30:1396-1401.

7. Frösen J, Piippo A, Paetau A, et al. Remodeling of saccular cerebral artery aneurysm wall is associated with rupture: histological analysis of 24 unruptured and 42 ruptured cases. Stroke 2004;35: 2287-2293.

8. Chyatte D, Bruno G, Desai $S$, et al. Inflammation and intracranial aneurysms. Neurosurgery 1999;45:1137-1146.

9. Tulamo R, Frösen J, Junnikkala $S$, et al. Complement activation associates with saccular cerebral artery aneurysm wall degeneration and rupture. Neurosurgery 2006;59:1069-1076.

10. Jayaraman T, Paget A, Shin YS, et al. TNF-alpha-mediated inflammation in cerebral aneurysms: a potential link to growth and rupture. Vasc Health Risk Manag 2008;4:805-817.

11. Frösen J, Piippo A, Paetau A, et al. Growth factor receptor expression and remodeling of saccular cerebral artery aneurysm walls: implications for biological therapy preventing rupture. Neurosurgery 2006;58:534-541.

12. Laaksamo $E$, Tulamo $R$, Baumann $M$, et al. Involvement of mitogenactivated protein kinase signaling in growth and rupture of human intracranial aneurysms. Stroke 2008;39:886-892.

13. Aoki T, Kataoka H, Shimamura M, et al. NF-kappaB is a key mediator of cerebral aneurysm formation. Circulation 2007;116:2830-2840.

14. Meri S, Pangburn MK. Discrimination between activators and nonactivators of the alternative pathway of complement: regulation via a sialic acid/polyanion binding site on factor $\mathrm{H}$. Proc Natl Acad Sci USA 1990;87:3982-3986.

15. Sim RB, Tsiftsoglou SA. Proteases of the complement system. Biochem Soc Trans 2004;32:21-27.

16. Sjöberg AP, Trouw LA, Blom AM. Complement activation and inhibition: a delicate balance. Trends Immunol 2009;30:83-90.

17. Biró A, Thielens NM, Cervenák L, et al. Modified low density lipoproteins differentially bind and activate the $\mathrm{C} 1$ complex of complement. Mol Immunol 2007;44:1169-1177.

18. Würzner $\mathrm{R}, \mathrm{Xu} \mathrm{H}$, Franzke $\mathrm{A}$, et al. Blood dendritic cells carry terminal complement complexes on their cell surface as detected by newly developed neoepitope-specific monoclonal antibodies. Immunology 1991;74:132-138.

19. Holvoet $\mathrm{P}$, Donck J, Landeloos $\mathrm{M}$, et al. Correlation between oxidized low density lipoproteins and von Willebrand factor in chronic renal failure. Thromb Haemost 1996;76:663-669.

20. Palinski W, Yla-Herttuala S, Rosenfeld ME, et al. Antisera and monoclonal antibodies specific for epitopes generated during oxidative modification of low density lipoprotein. Arteriosclerosis 1990;10:325-335.

21. Koistinen V, Wessberg S, Leikola J. Common binding region of complement factors $\mathrm{B}, \mathrm{H}$ and $\mathrm{CR} 1$ on $\mathrm{C} 3 \mathrm{~b}$ revealed by monoclonal anti-C3d. Complement Inflamm 1989;6:270-280.

22. Young SG, Witztum JL, Casal DC, et al. Conservation of the low density lipoprotein receptor-binding domain of apoprotein $\mathrm{B}$. Demonstration by a new monoclonal antibody, MB47. Arteriosclerosis 1986;6:178-188.

23. Zhang $M$, Alicot $E M$, Chiu I, et al. Identification of the target selfantigens in reperfusion injury. J Exp Med 2006;203:141-152.

24. Capella JF, Paik DC, Yin NX, et al. Complement activation and subclassification of tissue immunoglobulin $\mathrm{G}$ in the abdominal aortic aneurysm. J Surg Res 1996;65:31-33.

25. Oksjoki R, Kovanen PT, Mäyränpää MI, et al. Complement regulation in human atherosclerotic coronary lesions. Immunohistochemical evidence that $\mathrm{C} 4 \mathrm{~b}$-binding protein negatively regulates the classical complement pathway, and that $\mathrm{C} 5 \mathrm{~b}-9$ is formed via the alternative complement pathway. Atherosclerosis 2007;192:40-48.

26. Jamous MA, Nagahiro S, Kitazato KT, et al. Endothelial injury and inflammatory response induced by hemodynamic changes preceding intracranial aneurysm formation: experimental study in rats. J Neurosurg 2007;107:405-411.

27. Michel JB, Thaunat $O$, Houard $X$, et al. Topological determinants and consequences of adventitial responses to arterial wall injury. Arterioscler Thromb Vasc Biol 2007;27:1259-1268. 
28. Meuwissen M, van der Wal AC, Niessen HW, et al. Colocalisation of intraplaque $C$ reactive protein, complement, oxidised low density lipoprotein, and macrophages in stable and unstable angina and acute myocardial infarction. J Clin Pathol 2006;59: 196-201.

29. Frösen J. The Pathobiology of Saccular Cerebral Artery Aneurysm Rupture and Repair -a Clinicopathological and Experimental Approach. Helsinki University Press: Helsinki, 2006.

30. Reynolds GD, Vance RP. C-reactive protein immunohistochemical localization in normal and atherosclerotic human aortas. Arch Pathol Lab Med 1987;111:265-269.

31. Ji SR, Wu Y, Potempa LA, et al. Effect of modified C-reactive protein on complement activation: a possible complement regulatory role of modified or monomeric C-reactive protein in atherosclerotic lesions. Arterioscler Thromb Vasc Biol 2006;26:935-941.
32. Diehl EE, Haines III GK, Radosevich JA, et al. Immunohistochemical localization of modified C-reactive protein antigen in normal vascular tissue. Am J Med Sci 2000;319:79-83.

33. Davis III AE, Harrison RA, Lachmann PJ. Physiologic inactivation of fluid phase C3b: isolation and structural analysis of C3c, C3d,g (alpha 2D), and C3 g. J Immunol 1984;132:1960-1966.

34. Nakagawa $H$, Suzuki $S$, Haneda $M$, et al. Significance of glomerular deposition of C3c and C3d in IgA nephropathy. Am J Nephrol 2000;20:122-128.

35. Sakaki T, Kohmura E, Kishiguchi T, et al. Loss and apoptosis of smooth muscle cells in intracranial aneurysms. Studies with in situ DNA end labeling and antibody against single-stranded DNA. Acta Neurochir (Wien) 1997;139:469-474.

36. Pentimalli L, Modesti A, Vignati A, et al. Role of apoptosis in intracranial aneurysm rupture. J Neurosurg 2004;101:1018-1025. 